\title{
Resíduos de herbicidas em corpos hídricos - Uma revisão ${ }^{1}$
}

\author{
Herbicides residues in water bodies - A review
}

\author{
Edson Aparecido dos Santos ${ }^{2}$; Núbia Maria Correia ${ }^{3}$; Rafael Grossi Botelho ${ }^{4}$
}

\begin{abstract}
Resumo - Os herbicidas são os produtos fitossanitários mais utilizados no mundo e indispensáveis para a produção de alimentos em larga escala. São compostos sintetizados para ação tóxica contra plantas, porém, os mesmos podem apresentar efeitos negativos a diversos outros organismos. Uma das formas de contato e/ou absorção da molécula pelo organismo em questão, é quando a mesma encontra-se em um corpo hídrico. Dessa forma, monitorar a perda e acúmulo de herbicidas em mananciais é procedimento indispensável para produção agrícola sustentável, especialmente no Brasil, principal consumidor de produtos fitossanitários do mundo e um dos maiores produtores de alimentos e detentor de corpos hídricos. Propõem-se com essa revisão apresentar fatores técnicos, operacionais, edafoclimáticos, físicos e químicos dos herbicidas e legislativos a respeito da contaminação de mananciais por herbicidas. Também são apresentados herbicidas detectados recentemente em corpos hídricos do Brasil e as principais técnicas para detecção e quantificação dos produtos na água. Em função da importância do Brasil no cenário mundial, com relação à produção de alimentos, os estudos relacionados ao monitoramento de herbicidas em mananciais são considerados insatisfatórios, principalmente com relação a países que têm a agricultura desenvolvida há mais tempo.
\end{abstract}

Palavras-chaves: cromatografia, lixiviação, produtos fitossanitários, contaminação

Abstract - Herbicides are the most important pesticides in the world and essential for food production. They are synthesized to affect plants but on the other hand can cause negative impact to non-target organisms in case of contamination of aquatic environments. Thus, monitor the loss of herbicides to aquatic environments is an indispensable assignment to practice a sustainable agriculture, especially in Brazil: the most important pesticide user in the world, one of the largest food producers and watershed holder. The purpose of the text is to present technical factors, operational, edaphoclimatic, physical and chemical of the herbicides and laws about water sources contamination by it. In addition, results of works that monitored herbicides in Brazilians water bodies and the main techniques for detection and quantification of these products also will be presented. Due to the importance of Brazil on the world stage, with respect to food production, studies related to the monitoring of herbicides in water sources should be developed more frequently, especially with respect to countries that have agriculture developed over time.

Keywords: chromatography, leaching, pesticides, contamination

\footnotetext{
${ }^{1}$ Recebido para publicação em 15/12/2013 e aceito em 20/02/2014.

${ }^{2}$ Engenheiro Agrônomo. Pós-doutorando. PNPD-CAPES/Embrapa Milho e Sorgo. Rod. MG 424, Km 45, Sete Lagoas, MG, Brasil. E-mail: <edsonapsant@yahoo.com.br〉. (Autor para correspondência).

3 Professora Doutora. Departamento de Fitossanidade, Universidade Estadual Paulista (UNESP), Câmpus de Jaboticabal.

${ }^{4}$ Biólogo. Pós-doutorando. Centro de Energia Nuclear na Agricultura (CENA), Universidade de São Paulo (USP).
} 


\section{Introdução}

As plantas daninhas compõem o principal problema de defesa fitossanitária na agricultura mundial. A cada ano, o somatório das perdas, devido às diferentes formas de interferência nas culturas e ao efeito indireto das plantas à produção de alimentos, gira em torno de 95 bilhões de dólares (FAO, 2009). Nesse sentido, constantemente especialistas da área agrícola investem esforços em estudos com o propósito de avaliar diferentes métodos para manejo dessas plantas.

Os métodos contemplam práticas de prevenção à entrada de espécies no sistema, técnicas culturais, diferentes formas de controle físico ou mecânico e utilização de inimigos naturais. Porém, o controle mais utilizado é por meio da aplicação de herbicidas: produtos fitossanitários, formulados sinteticamente, que têm a função de inibir o desenvolvimento e/ou provocar a morte das plantas daninhas, quando utilizados em concentrações convenientes.

Em relação aos outros métodos de controle, o uso de herbicidas apresenta diversas vantagens no manejo de plantas daninhas e, de acordo com a evolução da agricultura, principalmente nas últimas décadas, observouse crescente número de produtos lançados, misturas, diferentes mecanismos de ação, épocas de aplicação, utilização de produtos várias vezes ao ano e o desenvolvimento de plantas geneticamente modificadas para não serem afetadas pelas moléculas dos produtos.

A substituição dos outros métodos de controle pela utilização de herbicidas faz com que o tempo gasto diminua em $97 \%$ durante o controle de plantas daninhas, fato que contribuiu para o crescimento da agricultura e o fornecimento de mão-de-obra para o desenvolvimento industrial de diversos países nas décadas passadas, bem como o aumento exponencial na oferta de alimentos (Gianessi, 2013). Por esses motivos, apenas no Brasil, maior consumidor de produtos fitossanitários atualmente, são registrados 540 herbicidas formulados, distribuídos em 142 ingredientes ativos (Brasil, 2013). Adicionalmente, dentre os produtos fitossanitários, a classe dos herbicidas é a mais utilizada no mundo.

Os herbicidas são, portanto, fundamentais para o desenvolvimento e estabilidade do agronegócio e, consequentemente, da economia de diversos países (CAI, 2008; Gianessi, 2013). Devido à dimensão de áreas utilizadas para produção de alimentos, somente no Brasil, são aplicados cerca de 470 mil toneladas de herbicidas anualmente, sendo 214 mil toneladas de ingredientes ativos (SINDAG, 2013). Considerando a área utilizada para agricultura, a aplicação média de herbicidas no Brasil é de $6,9 \mathrm{~kg} \mathrm{ha}^{-1} \mathrm{ano}^{-1}$ (IBGE, 2013).

Esse fato remete à preocupação com relação à segurança, à saúde do homem e ao meio ambiente como um todo, pois, durante a aplicação dos herbicidas, ocorrem perdas de 2 a 90\% (Taylor, 1995; Leu et al., 2004; Southwick et al., 2009). Além disso, os herbicidas, apesar de serem formulados com o propósito de ação contra plantas, podem também afetar direta ou indiretamente organismos de outros reinos, em função da toxicidade do ingrediente ativo e/ou das substâncias que compõem a formulação comercial. Diante de intoxicações a animais são citados danos em diversas enzimas, má constituição de tecidos, danos reprodutivos e comportamentais (Tsai, 2013).

Uma vez fora do seu local de ação, fato que é regra para a maior parte do produto fitossanitário aplicado, os herbicidas podem ser adsorvidos, absorvidos, sofrer degradação física, química ou biológica ou formar complexos com elementos do sistema (Flury, 1996). A grande variedade de fatores do ambiente, das práticas operacionais e dos produtos, adicionados à complexidade na relação entre eles, tornam difícil a predição de perdas de produtos fitossanitários no ambiente. No entanto, diante das diversas matrizes receptoras das moléculas, os corpos de água são os mais importantes acumuladores finais 
dos herbicidas, principalmente em agrossistemas com grande rede hidrográfica e alta intensidade de uso (Leu et al., 2004; Murray et al., 2010).

\section{Deslocamento dos herbicidas para os corpos hídricos}

Além da proximidade dos corpos de água da área tratada, diversos outros fatores estão relacionados à contaminação dos mananciais pelas moléculas. Esses fatores são ligados basicamente às características técnicas de aplicação, física e química dos produtos, solo, clima, topografia e ao manejo da área. Muitas vezes, um ponto negativo é integrado a outros e juntos otimizam o processo de chegada e permanência do herbicida na água.

Basicamente, a aplicação de herbicidas se dá utilizando-se a água como veículo aplicação líquida. No entanto, a chegada do produto ao manancial pode ocorrer antes da aplicação, pois muitas vezes os produtos, ou embalagens, são estocados ou manuseados em locais não indicados ou de forma incorreta. Esses locais, quando próximos a corpos de água, muitas vezes resultam na chegada do produto ao ambiente (Monquero et al., 2009; Tavella et al., 2011). As regras para armazenagem, manuseio de embalagens, dentre outras providências sobre os herbicidas, são determinadas de acordo com a Lei Federal $n^{\circ}$ 9.974 de 06 de junho de 2000 (que altera a Lei $\mathrm{n}^{\circ}$ 7.802).

Porém, apesar da importância do uso de produtos fitossanitários no Brasil, são constantemente relatados nos campos práticas de não obediência à Lei ou desatenção aos procedimentos com produtos formulados próximos a mananciais. Nesse sentido, Filizola et al. (2005), com o propósito de verificar a contaminação de córrego por defensivos agrícolas na região leste do Estado de São Paulo, verificaram a presença dos produtos na água e relacionaram o fato à lavagem de equipamentos e embalagens, próxima ao reservatório de água, e não ao uso dos produtos nas lavouras.

De fato, diante do risco de contaminação de mananciais, uma aplicação de herbicidas em campo, pode ser considerada insignificante em relação à lavagem de embalagens e equipamentos diretamente nos poços de água. Para exemplificação, considerase a aplicação de atrazine na dosagem de $2,0 \mathrm{~kg}$ $\mathrm{ha}^{-1}$. Durante a pulverização, na simulação do pior cenário de acordo com critérios de estudos ecotoxicológicos, onde o produto é aplicado diretamente no corpo de água, sem interação com material suspenso, ou com sedimentos, e diluição imediata e uniforme até 2,0 metros de profundidade (Urban \& Cook, 1986), seriam quantificados $100 \mu \mathrm{g} \mathrm{L}^{-1}$ de atrazine. Por outro lado, a lavagem de equipamentos e embalagens diretamente nos leitos dos córregos, promove a chegada direta de quilos dos produtos fitossanitários no manancial.

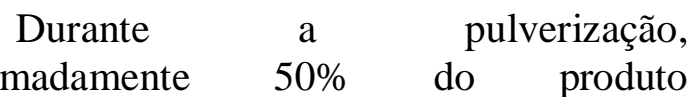
aproximadamente $50 \%$ do produto fitossanitário é perdido (Friedrich, 2004). Nesse sentido, é importante levar em consideração três parâmetros ambientais: ventos, umidade relativa e temperatura, que devem estar em consonância com características técnicas de aplicação, como pontas de pulverização, velocidade do aplicador, pressão de trabalho, alvo a ser tratado, condições do equipamento aplicador e qualificação técnica dos recursos humanos (Ferreira et al., 2007). Esse fator pode ser agravado em grandes áreas com culturas de elevado interesse agrícola com a possibilidade da aplicação aérea de herbicidas.

Integrando algumas das características supracitadas, Costa et al. (2007) ao realizarem a aplicação de marcador similar a um herbicida pré-emergente em solo, verificaram interação significativa entre a velocidade do vento, a ponta de pulverização e a pressão de trabalho nos resultados de deriva. Mas, observaram também que quanto maior a intensidade do vento, mais produto é perdido por arraste, e 
muitas vezes, a escolha da correta ponta de pulverização é melhor solução para contornar o efeito negativo do vento em relação à diminuição da pressão.

A ponta de pulverização é responsável pela formação e distribuição das gotas de pulverização. É considerada um item básico em um equipamento de aplicação de herbicidas, por estar diretamente relacionada às perdas do produto e, consequente, contaminação de mananciais. De acordo com Ferreira et al. (2007), pontas que produzem gotas com diâmetro menor que $100 \mu \mathrm{m}$ devem ser evitadas nas aplicações de herbicidas, pois a probabilidade de perdas é grande.

Nos campos de produção agrícola, além da perda do herbicida por deriva, pode haver também a chegada do mesmo ao solo e o transporte para o manancial por diferentes maneiras: escorrimentos superficial e subsuperficial e lixiviação. Inclusive, o potencial de lixiviação é a principal característica de um herbicida relacionada à contaminação de águas subterrâneas, de acordo com critérios elaborados pela Agência de Proteção Ambiental dos Estados Unidos (USEPA, 2013) e pelo índice GUS (Gustafon, 1989).

No mesmo sentido, o herbicida é classificado em relação à sua capacidade de contaminar águas superficiais de acordo com o método de Goss (Goss, 1992). Segundo esses critérios, a movimentação dos herbicidas no solo, e consequente classificação, é governada por características físico-químicas da molécula, conforme Tabela 1.

Tabela 1. Critérios para ranqueamento de produtos fitossanitários quanto ao potencial de contaminação de água subterrânea (EPA e GUS) e superficial (GOSS) e classificação de alguns herbicidas.

\begin{tabular}{|c|c|c|c|c|}
\hline Método & Características & \multicolumn{3}{|c|}{ Classes } \\
\hline USEPA $^{1}$ & $\begin{array}{l}\text { Solubilidade em água, coeficiente de } \\
\text { adsorção à matéria orgânica do solo, } \\
\text { constante da Lei de Henry, meia vida } \\
\text { em solo e água e Especiação. }\end{array}$ & \multicolumn{2}{|c|}{$\begin{array}{l}\text { Contaminante Potencial - CP } \\
\text { Contaminante - NC }\end{array}$} & Não \\
\hline GUS $^{2}$ & $\begin{array}{l}\text { Meia vida no solo e coeficiente de } \\
\text { adsorção à matéria orgânica. }\end{array}$ & \multirow{2}{*}{\multicolumn{3}{|c|}{$\begin{array}{l}\text { Não Lixiviável - NL }(\text { GUS < 1,8) } \\
\text { de Transição - FT }(1,8<\text { GUS < } 2,8) \\
\text { Provável Lixiviação - PL }(\text { GUS > 2,8) } \\
\text { Alto, Médio e Baixo Potencial de } \\
\text { Contaminação de águas superficiais, } \\
\text { Dissolvido em Água ou Associado ao } \\
\text { Sedimento (APCDA ou APCAS, MPCDA } \\
\text { ou MPCAS, BPCDA ou BPCAS) }\end{array}$}} \\
\hline $\mathrm{GOSS}^{3}$ & $\begin{array}{l}\text { Meia vida no solo, coeficiente de } \\
\text { adsorção à matéria orgânica do solo e } \\
\text { solubilidade em água. }\end{array}$ & & & \\
\hline \multirow{2}{*}{ Exemplos } & \multicolumn{4}{|c|}{ Classificação $^{4}$} \\
\hline & USEPA & GUS & GOS & \\
\hline $2,4-\mathrm{D}$ & $\mathrm{CP}$ & FT & APCAS e $\mathrm{N}$ & \\
\hline Ametryn & $\mathrm{NC}$ & FT & APCDA e & \\
\hline Atrazine & $\mathrm{CP}$ & PL & APCDA e I & \\
\hline Clomazone & $\mathrm{CP}$ & PL & APCDA e & \\
\hline Diuron & $\mathrm{CP}$ & PL & APCDA e 1 & \\
\hline Glyphosate & $\mathrm{CP}$ & NL & MPCDA e l & \\
\hline
\end{tabular}

Método escreening - Agência de Proteção Ambientel dos Estados Unidos, (USEPA, 2013). Também são considerados tipo de solo, posição do aquífero e precipitações. ${ }^{2}$ Groundwater Ubiquity Score - GUS (Gustafson, 1989). ${ }^{3}$ Método de GOSS (Goss, 1992). ${ }^{4}$ Adaptado de Milhome et al. (2009) e Britto et al. (2012). 
Adicionada às características relacionadas ao transporte, a persistência da molécula do herbicida no ambiente, na forma original, também é um fator diretamente relacionado à sua detecção em mananciais. Principalmente se há movimentação no sentido vertical do solo, uma vez que a degradação microbiana, considerada a principal forma de quebra das moléculas de herbicida no ambiente edáfico, é diminuída à medida que o herbicida se distancia da superfície. Além disso, o comportamento das moléculas no solo é influenciado por diversos outros fatores, tais como $\mathrm{pH}$, capacidade de troca catiônica, textura, mineralogia e teor de matéria orgânica, que determinam os processos de adsorção ou desorção do herbicida no solo (Vencill et al., 2002).

Nesse sentido, com o propósito de avaliar a movimentação e a persistência dos herbicidas sulfentrazone e imazapic, em função do tipo de solo e da precipitação, Monquero et al. (2010) verificaram que a intensidade de chuvas e a textura do solo estão diretamente relacionados ao transporte vertical dos herbicidas. Portanto, solos de textura média ou arenosos possibilitam maior movimentação dos produtos e, posterior, detecção em aquíferos, conforme também destacado por Gomes et al. (2006).

Outro fator relacionado à detecção de herbicidas em mananciais é o volume de produtos depositados em uma área. No Brasil, a cultura da soja até 2013 é a principal consumidora de herbicidas, em volume, e a cana-de-açúcar a segunda (SINDAG, 2013), porém, em função de características de manejo das duas culturas e dos herbicidas, produtos utilizados em cana-de-açúcar são mais comumente detectados em água com relação àqueles utilizados em soja. Dentre as características dos herbicidas utilizados nas duas culturas, a capacidade de persistência na forma original no solo é muito maior com herbicidas utilizados em canaviais, o que confere maior concentração de herbicidas em solos canavieiros. Mais de $70 \%$ dos herbicidas aplicados nessa cultura possuem ação residual (Vencill, 2002; Brasil, 2013), o que aumenta a probabilidade de perda para mananciais.

Algumas situações operacionais relacionadas às lavouras, como relevo, proteção e posição do manancial também influenciam na chegada dos herbicidas na água. Práticas de preparo do solo em locais com alta inclinação propiciam o carregamento de solo e herbicida para os córregos, principalmente em locais com solo de textura média ou arenosa e alta intensidade de chuvas. A esse respeito, Queiroz et al. (2011) ao avaliar a capacidade de lixiviação e o escorrimento superficial de glyphosate após simulação de chuvas, observaram que o produto é mais facilmente arrastado pela superfície. A capacidade de ligação a componentes da matéria orgânica do solo, óxidos de ferro, alumínio e argilas (Toni et al., 2006), faz com que a persistência do glyphosate no ambiente seja maior e que o mesmo continue sendo detectado em água 60 dias após a aplicação (Silva et al., 2003).

\section{Limite permitido de resíduos e metodologia para identificação e quantificação de herbicidas em água}

Com os propósitos de incentivar as boas práticas agrícolas e evitar impactos adversos da agricultura no meio ambiente e na saúde pública, grande parte dos países estabeleceu limites máximos permitidos para determinados herbicidas em amostras de água. Com o desenvolvimento de técnicas de quantificação mais sofisticadas e a constante preocupação da sociedade, em diversos países o número de moléculas com valores máximos permitidos aumentou e, em alguns casos, os limites tolerados foram reduzidos (Alder et al., 2006).

Uma das principais agências de proteção ambiental do mundo é situada nos Estados Unidos e rege as diretrizes sobre níveis de herbicidas permitidos em água naquele país. Essa entidade determina o limite máximo de 
diversos produtos fitossanitários em função de testes toxicológicos, da utilização e, consequente, ocorrência da molécula no manancial e do desenvolvimento do método analítico (USEPA, 2013).

Exemplificando, para o herbicida glyphosate, o limite máximo permitido em água potável nos Estados Unidos é de $700 \mu \mathrm{g}$ $\mathrm{L}^{-1}$, ao passo que para o 2,4-D e atrazine esse valor é de 70 e 3,0 $\mu \mathrm{g} \mathrm{L} \mathrm{L}^{-1}$, respectivamente. Nota-se, portanto, certa discrepância entre alguns valores adotados com relação às moléculas e também com relação a outros países. Pois, aqueles englobados pela União Européia definem que o limite máximo para qualquer produto fitossanitário em água de consumo humano, não pode exceder $0,1 \mu \mathrm{g} \mathrm{L}^{-1}$, e a soma de todas as moléculas detectadas e quantificadas não pode ultrapassar $0,5 \mu \mathrm{g} \mathrm{L}^{-1}$ (EUROPEAN UNION, 1998).

De acordo com a discrepância entre os limites máximos adotados nos diferentes países, há certa discussão pela carência de testes toxicológicos para suplantar os limites impostos pela União Européia, que se baseia no princípio da precaução e define o valor máximo de resíduo em função do limite de quantificação do método analítico. Entretanto, a Organização Mundial da Saúde (OMS) dita premissas para a definição de um limite máximo aceitável de determinado contaminante: i - há fortes evidências de contaminação da água pelo herbicida em questão; ii - o produto tem importância internacional ou iii - é integrante na lista de pesticidas avaliados pela OMS (OMS, 2004).

No Brasil, por meio da Resolução 357 do Conselho Nacional do Meio Ambiente, são estabelecidos limites máximos de produtos fitossanitários em água. No entanto, pouquíssimos herbicidas são mencionados na resolução, sendo eles: alachlor $\left(20,0 \mu \mathrm{g} \mathrm{L}^{-1}\right)$, atrazine $\left(2,0 \mu \mathrm{g} \mathrm{L}^{-1}\right), 2,4-\mathrm{D} \quad\left(4,0 \mu \mathrm{g} \mathrm{L}^{-1}\right)$, glyphosate $\left(65,0 \mu \mathrm{g} \mathrm{L}^{-1}\right)$, simazine $\left(2,0 \mu \mathrm{g} \mathrm{L}^{-1}\right)$ e trifluralin $\left(0,2 \mu \mathrm{g} \mathrm{L}^{-1}\right)$. Inclusive na lista de pesticidas avaliados pela OMS, poucos herbicidas utilizados no Brasil são citados (OMS, 2011).

Portanto, a capacidade em se detectar e quantificar com exatidão determinado herbicida em água, influencia diretamente na tomada de decisão das autoridades regulamentadoras diante da necessidade de reger os níveis de produto na água.

Basicamente, o monitoramento de resíduos de produtos fitossanitários em corpos de água é por meio de técnicas cromatográficas, que consistem em separar, identificar e quantificar espécies químicas. A cromatografia baseia-se na distribuição da amostra em duas fases, uma móvel e outra estacionária. Considerando o estado físico da fase móvel, distinguem-se a cromatografia gasosa (CG) e a cromatografia líquida (CL), nas quais as fases móveis podem ser um gás e um líquido, respectivamente (Collins \& Braga, 1987).

Ao longo dos anos, graças ao aperfeiçoamento e descobrimento de técnicas cromatográficas e de métodos de extração de amostras ambientais, tem sido possível determinar e quantificar produtos fitossanitários em concentrações cada vez menores. O número de moléculas avaliadas de forma simultânea também tem aumentado, de modo que que até 2013, os níveis quantificáveis em alguns métodos geram concentrações em ng $\mathrm{L}^{-1}$ e centenas de analitos podem ser avaliados simultaneamente (Gritti \& Guiochon, 2013).

Durante a evolução das técnicas cromatográficas (a partir dá década de 1970), foram observadas grandes mudanças. No princípio, a análise de resíduos de herbicidas era realizada principalmente por cromatografia gasosa com auxílio de detectores com captura de elétrons, nitrogênio e fósforo, ou por chama fotométrica. Posteriormente, por meio da integração com a espectrometria de massas, é possível determinar e confirmar (confiabilidade) a presença de determinado 
analito na amostra em função de sua massa molecular (Alder et al., 2006).

Destaca-se também, grande diferença entre a capacidade de análise em cromatografia líquida atual em relação às décadas passadas. Dentre as causas são citadas a baixa eficiência dos detectores de ultravioleta, arranjos de diodo e fluorescência com relação aos detectores, utilizados em cromatografia gasosa. No entanto, com o passar dos anos, o desenvolvimento do elétron-spray, assim como a ionização química à pressão atmosférica em conjunto com a espectrometria de massas, aumentou consideravelmente a sensibilidade durante a detecção por cromatografia líquida (Hernandez et al. 2005).

Em análise cromatográfica de herbicidas, sendo ela líquida ou gasosa, primeiramente é necessária a validação do método. De acordo com o Instituto Nacional de Metrologia, Normalização e Qualidade Industrial (INMETRO), os parâmetros levados em consideração nesse passo são: especificidade e seletividade, faixa de trabalho, linearidade, exatidão, precisão, limite de quantificação, limite de detecção e robustez. Abaixo são definidos os parâmetros (Brasil, 2011).

- Especificidade: o método produz resposta para apenas um analito;

- Seletividade: o método produz uma resposta para cada analito;

- Faixa de trabalho: Faixa de concentrações do analito, na matriz, no qual o método pode ser aplicado;

- Linearidade: Capacidade de um método em gerar resultados proporcionais às concentrações dos analitos nas amostras, dentro de uma determinada faixa de concentração conhecida (curva padrão). A linearidade se baseia no valor do coeficiente de correlação linear;

- Exatidão: é a medida de um valor próximo à do valor real;

- Precisão: é a reprodutibilidade de um resultado;
- Limite de quantificação: menor concentração de um determinado analito que o método consegue quantificar em uma amostra;

- Limite de detecção: menor concentração de um determinado analito que o método consegue detectar em uma amostra;

- Robustez: sensibilidade que o método apresenta frente a variações que possam ocorrer quando este está sendo executado.

Dentre os principais problemas técnicos em cromatografia, durante análise de herbicidas em amostras de água, destaca-se que algumas vezes é necessária pré-concentração do analito na amostra para posterior injeção da alíquota no aparelho. Após a concentração, há a necessidade de extração, porém, os métodos para tal etapa requerem o uso de uma grande quantidade de solventes orgânicos, reagentes e principalmente tempo. Diante disso, houve mudanças nos equipamentos com o intuito de aumentar a sensibilidade às moléculas desprezando a necessidade de extração quando se realiza a injeção direta da amostra.

Em águas subterrâneas, há menor quantidade de poluentes, o que facilita a técnica de injeção direta. A utilização de filtros, no preparo das amostras, também pode auxiliar e evitar contaminantes durante a corrida. No entanto, destaca-se que muitos componentes na amostra de água podem mascarar os resultados originais. Botelho et al. (2013) monitoraram resíduos de atrazine e ametryn durante 12 meses ao longo do rio Piracicaba (SP) e realizaram as análises por meio de injeção direta ao cromatógrafo em sistema de Cromatografia Líquida Acoplada à Espectrometria de Massas. Foram obtidos limites de quantificação e detecção iguais a 0,10 e $0,07 \mu \mathrm{g} \mathrm{L}^{-1}$, respectivamente, para atrazine, e 0,14 e $0,09 \mu \mathrm{g} \mathrm{L} \mathrm{L}^{-1}$ para ametryn. Huang et al. (2006) e Huang et al. (2008) também obtiveram baixos limites mínimos detectados quando realizaram a injeção direta das amostras.

$\mathrm{Na}$ análise de resíduos de produtos fitossanitários em matrizes ambientais, a 
escolha da técnica cromatográfica é extremamente importante e depende de três fatores básicos relacionados à molécula: volatilidade, polaridade e composição, que estão diretamente relacionadas à produção dos resultados. Nesse sentido, Alder et al. (2006) realizaram trabalho de revisão sobre análise de 500 produtos fitossanitários com o propósito de determinar a técnica mais sensível.

$\begin{array}{cccc}\text { Esses } & \text { autores } & \text { compararam } & \text { a } \\ \text { Cromatografia } & \text { Gasosa } & \text { Acoplada } & \text { à } \\ \text { Espectrometria } & \text { de Massas } & \text { (CG/MS) com } & \text { a } \\ \text { Cromatografia } & \text { Líquida } & \text { Acoplada } & \text { à } \\ \text { Espectrometria } & \text { de Massas } & \text { (LC-MS/MS) } & \text { e }\end{array}$
Espectrometria de Massas (LC-MS/MS) e observaram que a última apresentou melhores resultados em relação á sensibilidade e seletividade. Dos 500 produtos fitossanitários avaliados, 135 não foram detectados por CG/MS, ao passo que 47 não foram detectados por LC-MS/MS.

\section{Principais herbicidas quantificados em mananciais brasileiros}

Em termos de produção de alimentos, variedade e qualidade dos produtos colhidos, a agricultura brasileira é uma das mais importantes atualmente. O país é o maior produtor de cana-de-açúcar, café, laranja e frutas tropicais em geral, e acaba de conseguir o título de maior produtor de biodiesel. Adicionalmente, se destaca em diversas outras atividades agrícolas, como soja, milho e arroz e algodão, e tem o agronegócio crescente safra após safra, o que o classificará como maior produtor de alimentos nos próximos anos (FAO, 2013). Nesse sentido, devido à grande utilização de herbicidas e à preocupação com os possíveis efeitos dos resíduos desses produtos para o homem e para o meio ambiente, em recentes trabalhos tem-se notado a preocupação quanto à detecção das moléculas em mananciais.

Cerdeira et al. (2002), ao avaliarem a capacidade de movimentação do herbicida 2,4$\mathrm{D}$ e possível contaminação em área de recarga do aquífero Guaraní, verificaram, por meio de cromatografia a gás, que entre os anos de 1996 e 1999 esse herbicida não era contaminante do manancial em níveis iguais ou maiores que 0,1 $\mu \mathrm{g} \mathrm{L}^{-1}$. A pesquisa com 2,4-D se deu em função do grande uso do produto em cana-deaçúcar naquela época, na região amostrada. Quanto às suas características físicas e químicas, o 2,4-D (formulação amina) é pouco volátil, possui afinidade pela porção mineral do solo e apresenta baixos valores de meia vida no solo, colocando-o na faixa de transição (FT) na avaliação do índice de GUS (Gustafson, 1989) e ausente, pelo critério USEPA (USEPA, 2013).

Por outro lado, Gomes et al. (2001) constataram que o herbicida tebuthiuron, utilizado em cana-de-açúcar para manejar plantas daninhas em pré-emergência e com alto poder residual, foi detectado em todas as amostras de água coletadas, no período de 1995 a 1999, em 53 metros de profundidade, na mesma região onde Cerdeira et al. (2002) realizaram as avaliações nos anos de 1996 a 1999. Ressalta-se que o tebuthiuron possui meia vida aproximada de 360 dias em solo e coeficiente de sorção normalizado para o teor de carbono orgânico $\left(\mathrm{K}_{\mathrm{oc}}\right)$ de 80 dias, dados que auxiliam na classificação do produto como perigoso a água subterrânea ou superficial.

Em trabalhos recentes, Britto et al. (2012) classificaram os herbicidas diuron e ametryn como os mais propensos à contaminação de água superficial em relação a outros 12 produtos, por meio dos critérios propostos por USEPA e GUS. Nesse sentido, os autores verificaram que esses produtos estão presentes em rios no Estado de Sergipe em concentrações de até $0,9 \mu \mathrm{g} \mathrm{L}^{-1}$ (diuron) e 0,5 $\mu \mathrm{g} \quad \mathrm{L}^{-1} \quad$ (ametryn). Um dos principais contribuintes para a chegada dos produtos na água é a intensidade de chuva que gera escorrimento superficial de solo e herbicida (Britto et al., 2012).

O herbicida clomazone é registrado no Brasil para uso em soja, cana-de-açúcar, 
algodão, milho, arroz, batata, fumo, mandioca e pimentão (Brasil, 2013). Esse produto apresenta persistência superior a 150 dias no solo, é medianamente volátil, possui alta solubilidade em água e $\mathrm{K}_{\mathrm{oc}}$ igual a $300 \mathrm{~mL} \mathrm{~g}^{-1}$ (Rodrigues \& Almeida, 2011). Marchezan et al. (2007) e Silva et al. (2009) classificaram o clomazone como um dos mais importantes contaminantes de mananciais do Brasil, principalmente em cultivos de arroz. Segundo os autores, $50 \%$ das amostras de água coletadas possuíam clomazone em concentração máxima de 7,72 $\mu \mathrm{g} \mathrm{L}^{-1}$. De acordo com Marchezan et al. (2007), outros herbicidas utilizados na cultura do arroz, como quinclorac e propanil, também foram detectados em água superficial, em resíduo máximo de 6,6 e $12,9 \mu \mathrm{g} \mathrm{L}^{-1}$, respectivamente.

Em trabalho realizado no Estado do Mato Grosso, por meio da coleta de água superficial e subterrânea, Dores et al. (2006) relatam a presença dos herbicidas atrazine, metolachlor, metribuzin, simazine e trifluralin, nas concentrações máximas de $\left(\mathrm{em} \mu \mathrm{g} \mathrm{L}^{-1}\right)$ : 0,$156 ; \quad 1,732 ; \quad 0,351 ; 0,085$ e 0,102 , respectivamente. A esse respeito, Armas et al. (2007) detectaram os herbicidas acetochlor, ametryn, atrazine, clomazone, diuron, glyphosate hexazinone, isoxaflutole, pendimethalin, simazine, sulfentrazone, tebuthiuron e trifluralin em água superficial e sedimentos do Rio Corumbataí, região produtora de cana-de-açúcar no Estado de São Paulo. Os autores verificaram que as triazinas foram os maiores contaminantes, principalmente em função da persistência no solo e da solubilidade em água.

Em relação às triazinas, o atrazine é sem dúvida o herbicida de maior número de relatos como contaminante de água no mundo. No Brasil, nos estudos desenvolvidos por Armas et al. (2007), as concentrações do produto em água superficial variaram entre 0,6 e 2,7 $\mu \mathrm{g} \mathrm{L}^{-1}$, em águas coletadas em região com predomínio de cana-de-açúcar. Apesar do registro, o atrazine não é comumente utilizado no cultivo canavieiro, fator que destaca a capacidade de persistência do produto no solo e chegada à água de forma mais lenta.

O atrazine é classificado como muito suscetível à lixiviação, de acordo com os critérios USEPA, GUS e GOSS. A média de resíduo em corpos de água, em áreas de agricultura é de $20 \mu \mathrm{g} \mathrm{L}^{-1}$ (Nwani, 2010). Apesar de apresentar baixa pressão de vapor $\left(3,9 \times 10^{-5} \mathrm{mPa}\right)$, algumas características edafoclimáticas e a baixa adsorção ao solo contribuem para que esse herbicida seja encontrado em alta frequência, inclusive em água de chuvas (Moreira et al., 2012). Mas, a sua principal matriz é a água subterrânea (Rohr \& Mccoy, 2010). Porém, a ampla utilização pode ser considerada como a principal causa de ocorrência do atrazine nos mananciais, o que gerou a proibição de comercialização em alguns países, inclusive onde o herbicida ainda é detectado em água após 20 anos da proibição (Hillebrand et al., 2013).

Moreira et al. (2012) coletaram água superficial, subterrânea e de precipitações, no período de setembro de 2007 a outubro de 2009 no Estado do Paraná, e avaliaram a presença de 16 produtos fitossanitários. Dentre eles, o herbicida atrazine foi o mais importante e quantificado em concentração de $75,43 \mu \mathrm{g} \mathrm{L} \mathrm{L}^{-}$ 1; 9,33 $\mu \mathrm{g} \mathrm{L}^{-1}$ e $18,96 \mu \mathrm{g} \mathrm{L}^{-1}$, em água de chuva, rio e subterrânea, respectivamente.

Outros herbicidas têm sido constantemente pesquisados e detectados em mananciais hídricos no Brasil (Tabela 2). As pesquisas procuram esclarecer dúvidas da sociedade em relação aos resíduos, adotando-se parâmetros da legislação de outros países, como dos Estados Unidos da América e da Europa, por meio das suas respectivas agências de proteção ambiental. $\mathrm{O}$ desenvolvimento e acesso da comunidade científica aos meios de análises contribuem para os avanços nessa área e a realização de novos estudos. 
Tabela 2. Exemplos de herbicidas quantificados, em diferentes corpos hídricos, em campos agrícolas do Brasil.

\begin{tabular}{|c|c|c|c|c|c|}
\hline Herbicida & MRQ $^{1}$ & Matriz & Local & Época & Fonte \\
\hline 2,4-D & 74,5 & Córrego & Itajaí, SC & $12 / 2004$ a 03/2007 & $\begin{array}{c}\text { Pinheiro et al. } \\
\text { (2010) }\end{array}$ \\
\hline ametryn & 2,9 & Rio & Corumbataí, SP & $03 / 2004$ a 02/2005 & $\begin{array}{l}\text { Armas et al. } \\
\text { (2007) }\end{array}$ \\
\hline atrazine & 75,43 & Chuva & $\begin{array}{l}\text { Lucas do Rio } \\
\text { Verde, MT }\end{array}$ & 08/2007 a 04/2009 & $\begin{array}{l}\text { Moreira et al. } \\
\quad(2012)\end{array}$ \\
\hline clomazone & 7,72 & Rio & $\begin{array}{l}\text { Rio Grande do } \\
\text { Sul, RS }\end{array}$ & $12 / 1999$ a $03 / 2000$ & $\begin{array}{l}\text { Marchezan et } \\
\text { al. (2007) }\end{array}$ \\
\hline diuron & 0,9 & Rio & Aracajú, SE & 07/2009 a 07/2010 & $\begin{array}{l}\text { Britto et al. } \\
\text { (2012) }\end{array}$ \\
\hline glyphosate & 144 & $\begin{array}{l}\text { Água de arroz } \\
\text { irrigado }\end{array}$ & $\begin{array}{l}\text { Rio Grande do } \\
\text { Sul, RS }\end{array}$ & $\begin{array}{c}02 / 1999 ; 12 / 1999 \\
03 / 2000\end{array}$ & $\begin{array}{l}\text { Mattos et al. } \\
\quad(2002)\end{array}$ \\
\hline propanil & 12,9 & Rio & $\begin{array}{l}\text { Rio Grande do } \\
\text { Sul, RS }\end{array}$ & $12 / 1999$ a $03 / 2000$ & $\begin{array}{l}\text { Marchezan et } \\
\text { al. (2007) }\end{array}$ \\
\hline tebuthiuron & 0,33 & $\begin{array}{c}\text { Água } \\
\text { subterranea }\end{array}$ & $\begin{array}{c}\text { Ribeirao Preto, } \\
\text { SP }\end{array}$ & 03/2004 a 06/2006 & $\begin{array}{l}\text { Cerdeira et al. } \\
\text { (2007) }\end{array}$ \\
\hline trifluralin & 0,18 & Água potável & $\begin{array}{c}\text { Primavera do } \\
\text { Leste, MT }\end{array}$ & $\begin{array}{c}09 / 1998 ; 12 / 1998 \text { e } \\
04 / 1999\end{array}$ & $\begin{array}{l}\text { Dores et al. } \\
\quad(2008)\end{array}$ \\
\hline
\end{tabular}

${ }^{1}$ Máximo Resíduo Quantificado

\section{Considerações Finais}

Durante as práticas de produção de alimentos, fibras e madeira, a utilização de herbicidas é fundamental para manutenção do potencial produtivo das plantas. Entretanto, existe uma demanda crescente da sociedade em informações sobre os possíveis efeitos negativos quando ocorrem perdas desses produtos para mananciais. Esse fato gera atitudes dos órgãos gestores do meio ambiente, que, uma vez com as informações, podem definir nortes acerca da utilização do produto em questão.

As instituições de proteção aos recursos naturais, em países que têm a agricultura desenvolvida há mais tempo, possuem programas de monitoramento de corpos de água e gerenciam a presença e concentração de centenas de produtos fitossanitários. No Brasil, boa parte dos mais importantes herbicidas não tem os limites máximos permitidos fixados por qualquer regulamentação, e quando detectados em mananciais, normalmente, estão em concentrações acima dos critérios adotados pelos Estados Unidos e/ou países europeus. Dentre os herbicidas, alguns dos mais detectados e quantificados são: atrazine, clomazone, diuron, glyphosate e 2,4-D. Dentre os herbicidas, alguns dos mais detectados e quantificados em águas do Brasil são: atrazine, clomazone, diuron, glyphosate e 2,4-D.

Finalmente, espera-se que o acesso às informações, sobre a incidência de herbicidas em corpos hídricos pela sociedade, gere mudanças em procedimentos técnicos e legais acerca da comercialização e aplicação dos produtos, principalmente, devido à grande importância agrícola do Brasil.

\section{Referências}

ALDER, L. et al. Residue analysis of 500 high priority pesticides: better by GC-MS or LCMS/MS?. Mass Spectrometry Reviews, v.25, n.6, p.838-865, 2006.

ARMAS, E.D. et al. Diagnóstico espaçotemporal da ocorrência de herbicidas nas águas superficiais e sedimentos do Rio Corumbataí e 
principais afluentes. Química Nova, v.30, n.5, p. 1119-1127, 2007.

BOTELHO, R.G. Avaliação da qualidade da água do rio Piracicaba (SP) e efeito da vinhaça para organismos aquáticos antes e após a correção do pH. 2013. 107p. Tese (Doutorado em Ciências) - CENA, Universidade de São Paulo. Piracicaba, 2013.

BRASIL. Instituto Nacional de Metrologia, Normalização e Qualidade Industrial INMETRO. Orientações Sobre Validação de Métodos de Ensaios Químicos. DOQCGCRE-008. Revisão: 04 /2011. Disponível em: 〈http:// www.inmetro.gov.br>. Acesso em: 09/12/2013.

$\begin{array}{lrr}\text { BRASIL: } & \text { MINISTÉRIO } & \text { DA } \\ \text { AGRICULTURA, } & \text { PECUÁRIA } & \text { E }\end{array}$
ABASTECIMENTO. AGROFIT - Sistema de Agrotóxicos Fitossanitários. Disponível em: http://extranet.agricultura.gov.br/agrofit_cons/p rincipal_agrofit_cons. Acesso em: 15 de dezembro de 2013.

BRITTO, F.B. et al. Herbicidas no alto Rio Poxim, Sergipe e os riscos de contaminação dos recursos hídricos. Revista Ciência Agronômica, v.43, n.2, p.390-398, 2012.

CAI, D.W. Understand the role of chemical pesticides and prevent misuses of pesticides. Bulletin of Agricultural Science and Technology, v.1, n.6, p.36-38, 2008.

CERDEIRA, A.L. et al. Leaching and half-life of the herbicide tebuthiuron on a recharge area of Guarany aquifer in sugarcane fields in Brazil. Journal of Environmental Science and Health, B, v.42, n.21, p.635-639, 2007.

CERDEIRA, A.L. et al. Metodologia analítica de resíduo do herbicida 2,4-D (ácido 2,4diclorofenoxiacético) em amostras de água em área de cultivo de cana-de-açúcar. Pesticidas: Revista de Ecotoxicologia e Meio Ambiente, v.12, n.1, p.99-110, 2002.
COLLINS, C.H.; BRAGA, G.L. Introdução a métodos cromatográficos. Campinas: Editora Unicamp, 1987. 297p.

COSTA, A.G.F. et al. Efeito da intensidade do vento, da pressão e de pontas de pulverização na deriva de aplicações de herbicidas em préemergência. Planta Daninha, v.25, n.1, p.203210, 2007.

DORES, E.F.G.C. et al. Multiresidue determination of herbicides in environmental waters from Primavera do Leste Region (Middle West of Brazil) by SPE-GC-NPD. Journal of the Brazilian Chemical Society, v.17, n.5, p.866-873, 2006.

DORES, E.F.G.C. Pesticide Levels in Ground and SurfaceWaters of Primavera do Leste Region, Mato Grosso, Brazil. Journal of Chromatographic Science, v.46, n.12, p.585590, 2008.

EUROPEAN UNION. Directive 98/83/EC of the European Parliament and of the Council of 03 November 1998 relating to the quality of water intended for human consumption. Official Journal of the European Communities L 0083, 25/12/1998.

FAO - Food and Agriculture Organization of the United Nations. Dados de produção mundial. Disponível em: <http://faostat.fao.org/site/567/default.aspx\#an cor>. Acesso em: 14 de dezembro de 2013.

FAO - Food and Agriculture Organization of the United Nations. Dados de produção mundial. Disponível em: <http://faostat.fao.org/site/567/default.aspx\#an cor>. Acesso em: 14 de Setembro de 2013.

FERREIRA, L.R. et al. Tecnologia de aplicação de herbicidas. In: SILVA, A. A.; SILVA, J. F. (Eds.). Tópicos em manejo de plantas daninhas. Viçosa, MG: Universidade Federal de Viçosa, 2007. p.326-367.

FILIZOLA, H.F. et al. Monitoramento de agrotóxicos e qualidade das águas em área de 
agricultura irrigada. Revista Brasileira de Agrociência, v.11, n.2, p.245-250, 2005.

FLURY, M. Experimental evidence of transport of pesticides through field soils - a review. Journal of Environmental Quality, v.25, n.2, p.25-45, 1996.

FRIEDRICH, T. Quality in pesticide application technology. In: RAETANO, C.G.; ANTUNIASSI, U.R. Qualidade em tecnologia de aplicação. Botucatu: Fepaf, 2004. p.93-109.

GIANESSI, P.L. The Increasing Importance of Herbicides in Worldwide Crop Production. Pest Management Science, v.69, n.10, p.1099-1105, 2013.

GOMES, M.A.F. et al. Movimento do herbicida tebutiuron em dois solos representativos das áreas de recarga do aqǘfero Guarani. Revista Brasileira de Engenharia Agrícola e Amiental, v.10, n.2, p.479-483, 2006.

GOMES, M.A.F.; SPADOTTO, C.A.; LANCHOTTE, V.L. Ocorrência do herbicida tebuthiuron na água subterrânea da microbacia do Córrego do Espraiado, Ribeirão Preto - SP. Pesticidas: Revista de Ecotoxicologia e Meio Ambiente, v.11, n.4, p.65-76, 2001.

GOSS, D.W. Screening procedure for soils and pesticides for potential water quality impacts. Weed Technology, v.6, n.11, p.701-708, 1992.

GRITTI, F.; GUIOCHON, G. Perspectives on the Evolution of the Column Efficiency in Liquid Chromatography. Analytical Chemistry, v.85, n.6, p.3017-3035, 2013

GUSTAFSON, D.I. Groudwater ubiquity score: a simple method for assessing pesticide leachibility. Environmental Toxicology and Chemistry, v.8, n.4, p.339-357, 1989.

HERNANDEZ, F.; SANCHO, J.V.; POZO, O.J. Critical review of the application of liquid chromatography/mass spectrometry to the determination of pesticide residues in biological samples. Analytical and
Bioanalytical Chemistry, v.382, n.12, p.934946, 2005.

HILLEBRAND, O. et al. Chemograph analysis of two herbicides in a German karst aquifer. Geophysical Research Abstracts, Disponível em: http://meetingorganizer.copernicus.org/EGU20 13/EGU2013-1503.pdf. Acesso em: 14 de dezembro de 2013.

HUANG, S.B. et al. Direct Aqueous Injection Liquid Chromatography/Electrospray Ionization-mass Spectrometry/Mass Spectrometry Analysis of Water for Atrazine, Simazine, and Their Chlorotriazine Metabolites. Journal of Agricultural and Food Chemistry, v.54, n.12, p.713-719, 2006.

HUANG, S.B.; MAYER, T.J.; YORKLEY, R.A. Direct aqueous injection LC-ESI/MS/MS analysis of water for 11 chloro- and thiomethyltriazines and metolachlor and its ethanesulfonic and oxanilic acid degradates. Journal of Agricultural and Food Chemistry. v.56, n.11, p.2595-2602, 2008

IBGE. Instituto Brasileiro de Geografia e Estatística. Censo Agropecuário. Disponível em:

http://www.ibge.gov.br/home/estatistica/econo mia/agropecuaria/censoagro/. Acesso em: 19 de dezembro de 2013.

LEU, C. et al. Variability of herbicide losses from 13 fields to surface water within a small catchment after a controlled herbicide application. Environmental Science and Technology, v.38, n.14, p.3835-3841, 2004.

MARCHEZAN, E. et al. Rice herbicide monitoring in two brazilian river during the rice growing season. Scientia Agricola, v.64, n.6, p.131-137, 2007.

MATTOS, M.L.T. et al. Monitoramento ambiental do glifosato e do seu metabólito (ácido aminometilfosfônico) na água de lavoura de arroz irrigado. Revista de 
Ecotoxicologia e Meio Ambiente, v.2, n.4, p.145-154, 2002.

MILHOME, M.A.L. et al. Avaliação do potencial de contaminação de águas superficiais e subterrâneas por pesticidas aplicados na agricultura do Baixo Jaguaribe, CE. Engenharia Sanitária e Ambiental, v.14, n.3, p.363-372, 2009.

MONQUERO, P.A. et al. Lixiviação e persistência dos herbicidas sulfentrazone e imazapic. Planta daninha, v.28, n.1, p.185195, 2010.

MONQUERO, P.A.; INÁCIO, E.M.; SILVA, A.C. Levantamento de agrotóxicos e utilização de equipamento de proteção individual entre os agricultores da região de Araras. Arquivos do Instituto Biológico, v.76, n.1, p.135-139, 2009.

MOREIRA, J.C. et al. Contaminação de águas superficiais e de chuva por agrotóxicos em uma região do estado do Mato Grosso. Ciência e Saúde Coletiva, v.17, n.6, p.1557-1568, 2012.

MURRAY, K.E.; SHEEBA, M. T.; BODOUR, A.A. Prioritizing research for trace pollutants and emerging contaminants in the freshwater environment. Environmental Pollution, v.158, n.12, p.3462-3471, 2010.

NWANI, C.D. et al. Toxicity of the Herbicide Atrazine: Effects on Lipid Peroxidation and Activities of Antioxidant Enzymes in the Freshwater Fish Channa Punctatus (Bloch). International Journal of Environmental Research and Public Health, v.7, n.8, p.32983312, 2010.

OMS. Organização Mundial da Saúde. Guidelines for Drinking Water Quality: Recommendations. Chemical facts sheets, The fourth Edition. Geneva. 2011. 541p.

PINHEIRO, A. Presença de pesticidas em águas superficiais e subterrâneas na bacia do Itajaí, SC. Rega, v.7, n.2, p.17-26, 2010.
QUEIROZ, G.M.P. et al. Transporte de glifosato pelo escoamento superficial e por lixiviação em um solo agrícola. Química Nova, v.34, n.2, p.190-195, 2011.

RODRIGUES, B. N.; ALMEIDA, F. L. S. Guia de herbicidas. Londrina, $6^{a}$ ed., 2011. 697p.

ROHR, J.R.; McCOY, K.A. A Qualitative Meta-Analysis Reveals Consistent Effects of Atrazine on Freshwater Fish and Amphibians A Review. Environmental Health Perspectives, v.118, n.1, p.20-32, 2010.

SILVA, D.R.O. et al. Monitoramento de agrotóxicos em águas superficiais de regiões orizícolas no sul do Brasil. Ciencia Rural, v.39, n.9, p.2383-2389, 2009.

SILVA, M.D.; PERALBA, M.C.R.; MATTOS, M.L.T. Determinação de glifosato e ácido aminometilfosfônico em águas superficiais do arroio passo do pilão. Pesticidas: Revista de Ecotoxicoligia e Meio Ambiente, v.13, n.1, p.19-28, 2003.

SINDAG - Sindicato Nacional da Indústria de Produtos para Defesa Agrícola. Disponível em http://www.sindag.com.br/. Acesso em: 13 de Outubro de 2013.

SOUTHWICK, L.M.; APPELBOOM, T.W.; FOUSS, J.L. Runoff and leaching of metolachlor from Mississippi river alluvial soil during seasons of average and below-average rainfall. Journal of Agricultural and Food Chemistry, v.7, n.4, p.1413-1420, 2009.

TAVELLA, L.B. et al. O uso de agrotóxicos na agricultura e suas consequências toxicológicas e ambientais. Agropecuária Científica no Semiarido, v.7, n.2, p.6-12, 2011.

TAYLOR, A.W. The volatilization of pesticides residues. In: ROBERTS, T. R.; KEARNEY, P. C. Environmental behavior of agrochemicals. Chichester, UK: John Wiley \& Sons, 1995. p.257-306, 1995. 
TONI, L.R.M.; SANTANA, H.; ZAIA, D.A.M. Adsorção de glifosato sobre solos e minerais. Toxicology Environmental Chemistry, v.95, n.2, p.197-206, 2013.

TSAI, W.A review on environmental exposure and health risks of herbicide paraquat. Toxicology Environmental Chemistry, v.95, n.2, p.197-206, 2013.

URBAN, D.J.; COOK, N.J. Hazard Evaluation Division - Standard Evaluation Procedure Ecological Risk Assessment, U.S. EPA Publication 540/9-86-001, 1986.

USEPA. United States Environmental Protection Agency. Integrated Risk Information System. Disponível em: http://www.epa.gov/iris/index.html. Acesso em: 15 de dezembro de 2013.

VENCILL, V.K. Herbicide Handbook, 8th ed.; Weed Science Society of America: Lawrence, KS, 2002, 430 p. 